Journal for ImmunoTherapy of Cancer

\title{
LRP1B mutations are associated with favorable outcomes to immune checkpoint inhibitors across multiple cancer types
}

\author{
Landon C Brown (1) , ${ }^{1}$ Matthew D Tucker (D) , ${ }^{2}$ Ramy Sedhom, ${ }^{3}$ Eric B Schwartz, ${ }^{4}$ \\ Jason Zhu, ${ }^{5}$ Chester Kao, ${ }^{1}$ Matthew K Labriola (1) , ${ }^{1}$ Rajan T Gupta, ${ }^{1}$ \\ Daniele Marin, ${ }^{1}$ Yuan Wu, Santosh Gupta, ${ }^{1}$ Tian Zhang (D) , ${ }^{1}$ Michael R Harrison, ${ }^{1}$ \\ Daniel J George, ${ }^{1}$ Ajjai Alva, ${ }^{4}$ Emmanuel S Antonarakis, ${ }^{3}$ Andrew J Armstrong ${ }^{1}$
}

To cite: Brown LC, Tucker MD, Sedhom $\mathrm{R}$, et al. $L R P 1 B$ mutations are associated with favorable outcomes to immune checkpoint inhibitors across multiple cancer types. Journal for ImmunoTherapy of Cancer 2021;9:e001792. doi:10.1136/ jitc-2020-001792

These data were presented at the ASCO annual meeting 2020, Abstract \# 3007.

Accepted 14 January 2021

Check for updates

(c) Author(s) (or their employer(s)) 2021. Re-use permitted under CC BY-NC. No commercial re-use. See rights and permissions. Published by BMJ.

For numbered affiliations see end of article.

Correspondence to Dr Andrew J Armstrong; Andrew.armstrong@duke.edu

\section{ABSTRACT}

Background Low-density lipoprotein receptor-related protein $1 \mathrm{~b}$ (encoded by $L R P 1 B$ ) is a putative tumor suppressor, and preliminary evidence suggests $\angle R P 1 B$ mutated cancers may have improved outcomes with immune checkpoint inhibitors (ICI).

Methods We conducted a multicenter, retrospective pancancer analysis of patients with $\angle R P 1 B$ alterations treated with ICl at Duke University, Johns Hopkins University (JHU) and University of Michigan (UM). The primary objective was to assess the association between overall response rate $(\mathrm{ORR})$ to $\mathrm{ICl}$ and pathogenic or likely pathogenic $(\mathrm{P} /$ $\mathrm{LP}) \angle R P 1 B$ alterations compared with $\angle R P 1 B$ variants of unknown significance (VUS). Secondary outcomes were the associations with progression-free survival (PFS) and overall survival (OS) by $\angle R P 1 B$ status.

Results We identified 101 patients (44 Duke, $35 \mathrm{JHU}, 22$ UM) with $L R P 1 B$ alterations who were treated with $I C l$. The most common tumor types by alteration (P/LP vs VUS\%) were lung ( $36 \%$ vs $49 \%)$, prostate $(9 \%$ vs $7 \%)$, sarcoma ( $5 \%$ vs $7 \%$ ), melanoma ( $9 \%$ vs $0 \%$ ) and breast cancer ( $3 \%$ vs $7 \%$ ). The 0 RR for patients with $L R P 1 B P / L P$ versus VUS alterations was $54 \%$ and $13 \%$, respectively (OR 7.5 , $95 \% \mathrm{Cl} 2.9$ to 22.3, $\mathrm{p}=0.0009)$. P/LP LRP1B alterations were associated with longer PFS (HR $0.42,95 \% \mathrm{Cl} 0.26$ to $0.68, p=0.0003$ ) and $0 S$ (HR $0.62,95 \% \mathrm{Cl} 0.39$ to $1.01, p=0.053)$. These results remained consistent when excluding patients harboring microsatellite instability (MSI) and controlling for tumor mutational burden (TMB).

Conclusions This multicenter study shows significantly better outcomes with ICI therapy in patients harboring $\mathrm{P} /$ $L P$ versus VUS $L R P 1 B$ alterations, independently of TMB/ MSI status. Further mechanistic and prospective validation studies are warranted.

\section{BACKGROUND}

The encouraging success of the immune checkpoint inhibitors (ICI) has dramatically improved the care of patients in a growing number or cancer subtypes. These therapies have become first-line options for many cancers, including melanoma, non-small cell lung cancer (NSCLC) and renal cell carcinoma. ${ }^{1-3}$ However, not all patients respond to treatment with ICIs. The use of predictive biomarkers for response has been explored in many tumor types with varying degrees of success. Programmed-death ligand-1 (PDL1) expression, ${ }^{1}$ microsatellite instability (MSI)/mismatch repair deficiency ${ }^{4}$ and tumor mutational burden (TMB $)^{56}$ are the only FDA-approved predictive biomarkers for immunotherapy response. Other potential biomarker candidates include tumor infiltrating lymphocytes, ${ }^{7}$ immunophenotyping, ${ }^{8}$ inflamed gene expression profiling ${ }^{9}$ and the gut microbiome. ${ }^{10}$

One potential predictive biomarker candidate is the low-density lipoprotein receptorrelated protein $1 \mathrm{~b}$, encoded by the $L R P 1 B$ gene. $L R P 1 B$ is a large gene located on chromosome $2 q$, containing $>91$ exons and spanning over 500 kilobases, and is a member of the LDL receptor family. ${ }^{11}$ The protein product of $L R P 1 B$ is 4599 amino acids long. Liu et al demonstrated that $50 \%$ of NSCLC cell lines harbored alterations of the $L R P 1 B$ gene (complete or partial homozygous deletions), and implicated $L R P 1 B$ as a likely putative tumor suppressor. ${ }^{12}$ Subsequent investigation has shown that $L R P 1 B$ may be a tumor suppressor in gastric cancer, where it is regulated by methylation. ${ }^{13}$ In addition, $L R P 1 B$ is altered or inactivated in many other solid tumors and hematological malignancies. $^{13-27}$ In a study of 3312 human cancer specimens, $L R P 1 B$ was one of the top 10 most frequently deleted genes. ${ }^{28}$ Based on data from The Cancer Genome Atlas data available on the cBioPortal database, the frequency of somatic $L R P 1 B$ alteration was $11.8 \%$ across all samples and $>20 \%$ of cases of NSCLC, 
melanoma, esophageal, stomach, head and neck, uterine and bladder cancers. ${ }^{29} 30$

A number of studies have suggested a correlation between $L R P 1 B$ alterations and improved outcomes with ICI. ${ }^{31-36}$ It remains to be determined if $L R P 1 B$ alterations are simply a prognostic passenger biomarker of high $\mathrm{TMB}$, or whether $L R P 1 B$ may have true biologic relevance and can independently predict responses to ICI. Thus, we performed a multi-institutional retrospective study to describe the outcomes of patients across multiple tumor types harboring alterations in $L R P 1 B$ who were treated with ICI, and to compare outcomes in patients with pathogenic/likely pathogenic (P/LP) and variant of unknown significance (VUS) LRP1B alterations.

\section{METHODS}

\section{Patients and eligibility}

We performed a retrospective review of all patients with $L R P 1 B$ alterations reported on tissue-based nextgeneration sequencing (NGS) panels who also received ICI at the Duke Cancer Center between July 2015 and October 2018, Johns Hopkins University (JHU) from May 2013 to September 2019 and University of Michigan (UM) from July 2013 to January 2020.

All patients with advanced or metastatic malignancies and a $L R P 1 B$ alteration were eligible. Patients who received chemotherapy concurrently with ICI were excluded, and patients treated in the neoadjuvant/adjuvant setting were also excluded. Patients at Duke and JHU were identified as having $L R P 1 B$ alterations from a genomic database, and ICI receipt was identified on chart review to generate the final patient list. At UM, patients were identified from an immunotherapy patient database, which was cross-referenced with patients who had tissue-based NGS.

\section{Tissue-based NGS}

Tissue-based NGS at Duke and JHU was performed on the Foundation Medicine platform. All Foundation Medicine panels used for this analysis were prior to the currently available CDX panel; at the time of this writing, $L R P 1 B$ is not included on the FoundationOne CDX panel. Tissue-based NGS at UM was performed on an in-house NGS panel, Michigan Oncology Sequencing Center(MIONCOSEQ). LRP1B alterations were considered in three groups. $\mathrm{P}$ alterations were defined as any genomic alteration that would lead to a large deletion, truncation or loss of function (ie, nonsense mutation, homozygous loss, frameshift mutation, intragenic rearrangement, splice acceptor/donor mutation). Missense mutations were then further categorized as LP alterations if they were listed in the Catalogue of Somatic Mutations in Cancer (COSMIC) database with a Functional Analysis through Hidden Markov Models (FATHMM) score for likelihood of pathogenicity of $>0.5 .{ }^{3738}$ VUS were defined as missense changes not listed in COSMIC or having FATHMM scores of $<0.5$.

\section{Patient variables}

The variables collected included patient demographics (age, race, ethnicity), tumor type (lung, kidney, bladder and so on), treatment selection (pembrolizumab, nivolumab and so on), PD-L1 status and TMB. Patient outcomes included best radiographic response, progression-free survival (PFS) and overall survival (OS). On patients collected at Duke and JHU, best radiological response was characterized by RECIST V.1.1 criteria. $^{39}$ At UM, best radiological response was determined using RECIST V.1.1 definitions in combination with clinical notes.

\section{Statistical analysis}

The primary outcome was the difference in overall response rates (ORR, complete or partial response) between the P/LP LRP1B alterations compared with the $L R P 1 B$ VUS subgroup. Other secondary outcomes included PFS, defined as time from initiation of ICI to progression or death (progression was defined by radiographic criteria or clear clinical progression based on chart review of notes from the treating physician) and OS. Logistic regression was used to compare ORR between the P/LP and VUS subgroups using a prespecified twosided alpha error of 0.05. The Cox proportional hazard model was used for time-to-event outcomes such as OS and PFS between the P/LP and VUS subgroups, using an alpha error of 0.05 for nominal significance testing between groups along with 95\% CIs. Logistic regression and Cox proportional hazard models were used for post hoc subgroup analysis between different institutions as well as between tumor types. No formal sample size calculation was performed, as all cases across three institutions were included. With a final sample size of 101 patients (45 VUS and $56 \mathrm{P} / \mathrm{LP}$ alterations), and assuming an ORR of $25 \%$, a post hoc calculation shows our study has $80 \%$ power to detect an ORR in the P/LP category of $54.2 \%$ or an OR of 3.55.

An additional analysis was performed to adjust for MSI status and TMB in which all patients with MSI-high (MSI-H) or MSI-unavailable status were excluded and then ORs for overall response and HR for PFS and OS were adjusted for TMB as a continuous variable as well as a binary variable (TMB $>10 \mathrm{vs}<10 \mathrm{mut} / \mathrm{Mb}$ ). To compare the rate of TMB between genomic alteration groups, the Wilcoxon rank-sum test was used.

\section{RESULTS \\ Patients}

A total of 101 patients were identified who harbored alterations in $L R P 1 B$ and received therapy with ICI. Duke, JHU and UM identified 44, 35 and 22 patients, respectively (see Consolidated Standards of Reporting Trials diagram, figure 1A). Demographics are shown in table 1 according to $L R P 1 B$ alterations. The $\mathrm{P} / \mathrm{LP}$ are combined for comparison against the $L R P 1 B$ VUS internal control group. The distribution of missense mutations for LP and 


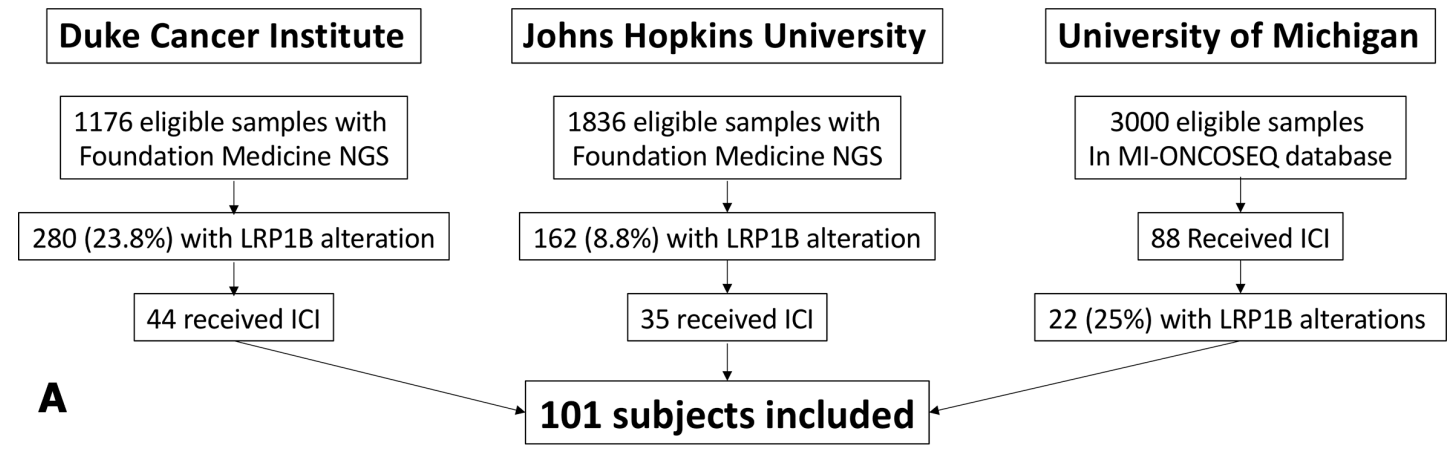

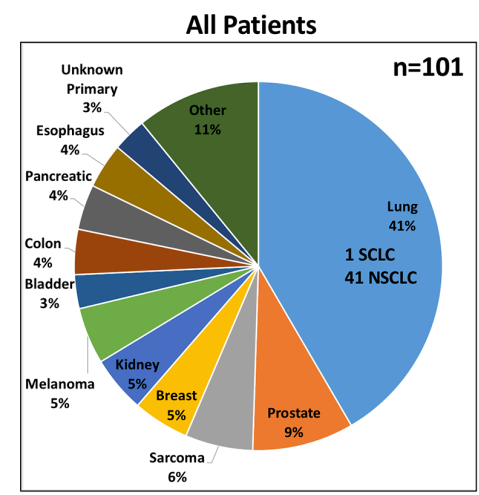

Other: Non-hodgkin lymphoma (1), Hodgkin lymphom (1), cholangiocarcinoma (1), brain (1) uterus (1), anal

\section{B}

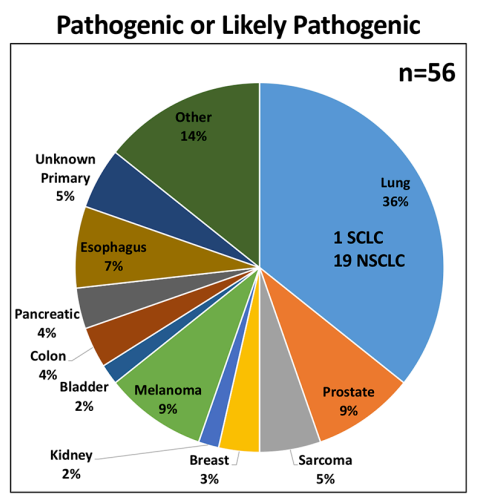

Other: Non-hodgkin lymphoma (1), uterus (1), anal (1), rectal (1), head and neck SCC(1), cutaneous SCC(3)

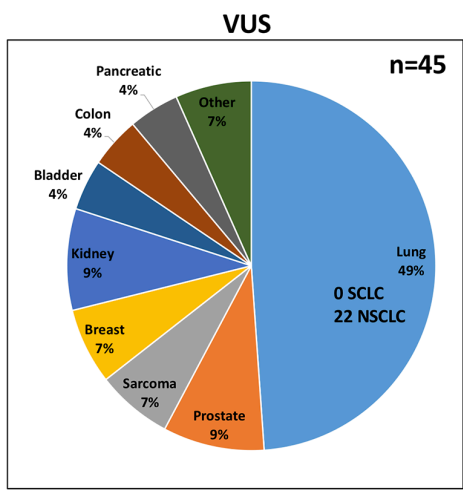

Other: Hodgkin lymphoma (1), cholangiocarcinoma (1) brain (1)

Figure 1 (A) Consolidated Standards of Reporting Trials diagram. (B) Distribution of malignancies identified for inclusion. ICI, immune checkpoint inhibitor; LRP1B, lipoprotein receptor-related protein 1b; NGS, next-generation sequencing; SCLC, small cell lung cancer; NSCLC, non-small cell lung cancer; SCC, squamous cell carcinoma.

Table 1 Demographics

\begin{tabular}{|c|c|c|c|}
\hline & All patients $n=101$ & $\begin{array}{l}\text { Pathogenic or likely } \\
\text { pathogenic } n=56\end{array}$ & VUS $n=45$ \\
\hline Male (\%) & $60 \%$ & $68 \%$ & $51 \%$ \\
\hline \multicolumn{4}{|l|}{ ICI type } \\
\hline Anti-PD-(L) $1^{*}$ & $91 \%$ & $88 \%$ & $96 \%$ \\
\hline \multicolumn{4}{|l|}{ Microsatellite instability } \\
\hline MSI-H & $8 \%$ & $9 \%$ & $7 \%$ \\
\hline MSS & $79 \%$ & $88 \%$ & $69 \%$ \\
\hline Not available & $13 \%$ & $3 \%$ & $24 \%$ \\
\hline Not available & $73 \%$ & $68 \%$ & $80 \%$ \\
\hline
\end{tabular}

${ }^{*} 61 \%$ pembrolizumab, 32\% nivolumab, 3\% atezolizumab, 3\% durvalumab, $1 \%$ cemiplimab.

CTLA-4, cytotoxic T-lymphocyte-associated protein 4; MSI-H, microsatellite instability-high; MSS, microsatellite stable; PD-L1, programed death receptor ligand 1; VUS, variants of unknown significance. 
VUS groups are shown in online supplemental figure 1 . The most common ICI therapy was single-agent antiPD-1 or anti-PD-L1 at 91\% (61\% of which was pembrolizumab and $32 \%$ nivolumab). Combination anti-PD-1 or anti-PD-L1 plus anticytotoxic T-lymphocyte-associated protein 4 (anti-CTLA-4) (primarily nivolumab and ipilimumab) was administered to $8 \%$ of subjects, which was over-represented in the P/LP group at $13 \%$ compared with $2 \%$ in the VUS group. Median time on therapy for all patients, P/LP cohort and VUS cohort was 4.1, 4.4 and 3.4 months, respectively. Median follow-up for all patients, P/LP cohort and VUS cohort was 10.3, 8.5 and 13.5 months, respectively.

Other notable differences between the $\mathrm{P} / \mathrm{LP}$ and VUS groups include a higher proportion of MSI-H (9\% vs $5 \%$ ) and PD-L1 >1\% (25\% vs $18 \%$ ) in the P/LP group. Notably, PD-L1 information was not available in a majority of patients $(72 \%)$. The cancer type is shown in figure 1B for the entire cohort and each molecular subgroup. Lung cancer was the most common tumor type (41\%, 40/41 NSCLC, $1 / 41$ small cell lung cancer) followed by prostate cancer $(9 \%)$, sarcoma $(6 \%)$, breast cancer (5\%), kidney cancer $(5 \%)$ and melanoma (5\%). Notable differences between the P/LP and VUS groups include imbalances in the rates of lung cancer ( $36 \%$ vs $49 \%$ ), melanoma (9\% vs $0 \%$ ), esophagus ( $7 \%$ vs $0 \%)$, unknown primary ( $5 \%$ vs $0 \%$, cutaneous or head and neck squamous cell carcinoma ( $7 \%$ vs $0 \%$ ), and kidney ( $2 \%$ vs $9 \%$ ) across $L R P 1 B$ alteration groups.

\section{Patient outcomes}

The primary outcome of radiographic response rates by molecular subtype are shown in figure 2 and table 2. The ORR (CR+PR) in the P/LP group was $30 / 56$ (54\%; $95 \%$ CI $40 \%$ to $67 \%$ ) compared with $6 / 45$ (13\%; $95 \%$ CI $5 \%$ to $27 \%$ ) in the VUS group. The OR by logistic regression for the ORR for $\mathrm{P} / \mathrm{LP}$ versus VUS was significant at 7.5 (95\% CI 2.9 to 22.3 , $\mathrm{p}=0.0009$ ).

PFS and OS are shown in table 3, with Kaplan-Meier plots shown in figure 3 and online supplemental figure 4. The P/LP group showed a significantly improved PFS compared with the VUS group by Cox proportional hazard analysis (HR 0.42; 95\% CI 0.26 to $0.68, \mathrm{p}=0.0003$ ) and median PFS of 8.4 months (95\% CI 5.2 to 23 ) vs 3.4 months (95\% CI 2.8 to 4.3 ), respectively. The P/LP group also showed an improvement in OS compared with the VUS group (HR 0.62; 95\% CI 0.39 to $1.01, \mathrm{p}=0.053$ ) with median PFS of 15.5 months (95\% CI 10.3 to 25.1 ) vs 9.5 months (95\% CI 7.6 to 21.3), respectively. In total, there were 77 PFS events and 69 deaths in the entire cohort. The $\mathrm{P} / \mathrm{LP}$ and VUS cohorts experienced 37 and $40 \mathrm{PFS}$ events, respectively. The P/LP and VUS cohorts experienced 34 and 35 deaths, respectively.

For a more comprehensive view of patient-level timeto-event data, a swimmer's plot of all patients separated by molecular subtype (P/LP vs VUS) showing best radiographic response, treatment duration, progression and death is shown in online supplemental figure 2.

\section{Best Overall Response}

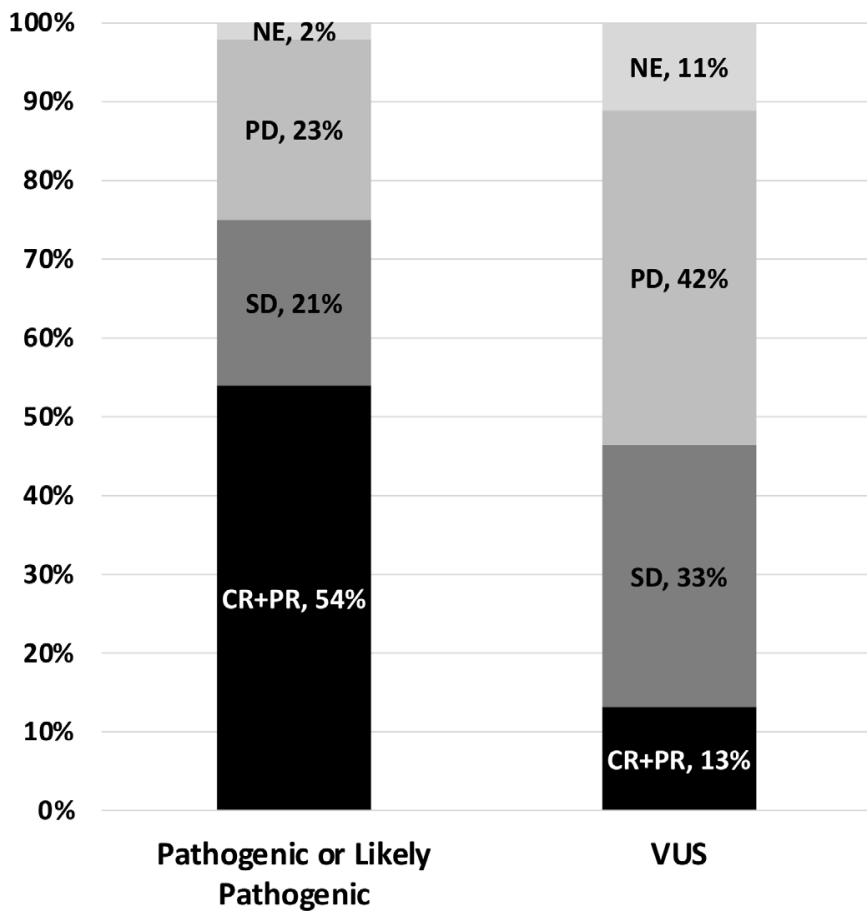

Figure 2 Stacked bar graph of best overall response among molecular subtypes. CR, complete response; NE, not evaluable; PD, progressive disease; PR, partial response; SD, stable disease; VUS, variants of unknown significance.

\section{Association with tumor mutational burden}

Box-and-whisker plots for TMB are shown in online supplemental figure 3. Median TMB in the P/LP group was $10 \mathrm{mut} / \mathrm{Mb}$ vs $7.9 \mathrm{mut} / \mathrm{Mb}$ in the VUS group. By the Wilcoxon rank-sum test, the two groups were not different $(\mathrm{p}=0.32)$. The $\mathrm{P} / \mathrm{LP}$ group showed more outliers with TMB $>50$ mut $/ \mathrm{Mb}$ at 13 patients (23.2\%) compared with the VUS group with three patients (6.7\%). MSI-H was identified in 5/56 (8.9\%) in P/LP group and $3 / 45$ $(6.7 \%)$ in the VUS group.

\section{Subgroup analysis}

Shown in figure 4 are forest plots of ORs for overall response and HRs for OS and PFS across institutions and cancer subtypes (lung cancer and non-lung cancers). In the unadjusted analysis, ORs and HRs remained consistent in favor of the P/LP group across the three institutions. Overall response and PFS also were consistent in the unadjusted analysis across patients with lung cancer and those without lung cancer. The OS benefit in the $\mathrm{P} /$ LP versus VUS group appeared to be strongest and most significant in patients with lung cancer, HR 0.41 (95\% CI 0.19 to 0.91 ), but not among those with non-lung cancers, HR 0.82 (95\% CI 0.44 to 1.51 ).

Two adjusted analysis were performed to isolate the contribution of TMB and MSI status, as shown in figure 4. All patients who were MSI-H $(n=8)$ or MSI-unavailable $(n=13)$ or missing TMB data $(n=8)$ were excluded, and then subsequently the three analysis of overall response, PFS and OS were adjusted by TMB in two fashions, as a 
Table 2 Best overall response and overall response rate among molecular subtypes

Pathogenic or likely pathogenic

$\mathrm{n}=56$

\begin{tabular}{lll}
\hline Best response, $\mathrm{n}(\%)$ & $2(4 \%)$ & $1(2 \%)$ \\
\hline Complete response (CR) & $28(50 \%)$ & $5(11 \%)$ \\
Partial response (PR) & $12(21 \%)$ & $15(33 \%)$ \\
Stable disease & $13(23 \%)$ & $5(11 \%)$ \\
Progressive disease & $1(1.8 \%)$ & $6(13 \%)(5 \%$ to $27 \%)$ \\
Not evaluable & $30(54 \%)(40 \%$ to $67 \%)$ & Reference $(1.0)$ \\
Overall response $(\mathrm{CR}+\mathrm{PR}), \mathrm{n}(\%)(95 \% \mathrm{Cl})$ & $7.5(2.9$ to 22.3$), \mathrm{p}=0.0009$ & \\
\hline OR of CR+PR $(95 \% \mathrm{Cl})$ & & \\
\hline
\end{tabular}

VUS, variants of unknown significance.

continuous variable and as a binary variable using the cutoff of $10 \mathrm{mut} / \mathrm{Mb}$ on a subset of patients based on established cutoffs from published clinical trials in NSCLC. ${ }^{40} 41$ This analysis included 75 patients ( 3 patients were missing both TMB and MSI information). Our overall findings remained consistent across TMB as a continuous or binary variable. When controlling for TMB as a continuous variable, the OR for overall response was 6.75 (95\% CI 2.32 to 23.00) and HRs for PFS and OS were 0.49 (95\% CI 0.28 to 0.87 ) and 0.77 (95\% CI 0.42 to 1.39 ), respectively. Similar to the unadjusted analysis for OS, the TMB-adjusted analysis for OS, but not overall response or PFS, showed that the adjusted OS benefit for the P/LP group over the VUS group appeared to driven by patients with lung cancer HR 0.34 (95\% CI 0.12 to 0.94 ) as compared with those with non-lung cancers, 1.31 (95\% CI 0.58 to 2.99).

An imbalance in the patients who received CTLA-4, primarily in combination with anti-PD-1 therapy inhibitors, was seen with a higher proportion of patients receiving anti-CTLA-4 therapy in the P/LP compared with the VUS group. To isolate the contribution of anti-CTLA- 4 therapy, an analysis was performed excluding two patients in VUS group (one who received anti-CTLA-4 monotherapy and one who received combination anti-PD-1/anti-CTLA-4) and excluding seven patients in the P/LP group (all who received anti-PD-1/anti-CTLA-4). In this subset analysis, ORR continued to favor P/LP over VUS for the entire cohort $49 \%$ (95\% CI 35 to 63 ) vs $14 \%$ (95\% CI 4 to 24$)$, respectively, for an OR of 5.9 (95\% CI 2.2 to 17.9), and $53 \%(95 \%$ CI 30 to 75$)$ vs $14 \%$ (95\% CI 0 to 28 ), respectively, among patients with lung cancer for an OR of 7.0 (95\% CI 1.4 to 25.9). This relationship was consistent in both the unadjusted analysis and when adjusting for TMB and excluding MSI-H (data not shown). Similarly, PFS continued to favor the LP/P group over VUS for all patients (HR $0.49,95 \%$ CI 0.30 to 0.81 ) and patients with lung cancer (HR $0.47,95 \%$ CI 0.22 to 1.0 ). OS was also numerically similar, but with a wider CI when excluding patients receiving anti-CTLA-4 in the entire cohort (HR $0.72,95 \%$ CI 0.44 to 1.2 ) and for patients with lung cancer (HR $0.44,95 \%$ CI 0.20 to 0.96 ). The HRs for OS and PFS

Table 3 Progression-free survival (PFS) and overall survival (OS) between patients with pathogenic or likely pathogenic (P/LP) alterations compared with those with VUS

\begin{tabular}{|c|c|c|}
\hline & $P / L P * n=46$ & VUS $n=55$ \\
\hline Median PFS, months (95\% Cl) & 8.4 (5.2 to 23$)$ & 3.4 (2.8 to 4.3$)$ \\
\hline 6-month PFS $(95 \% \mathrm{Cl}) \dagger$ & 0.59 (0.47 to 0.73$)$ & $0.27(0.17$ to 0.45$)$ \\
\hline 12-month PFS $(95 \% \mathrm{Cl}) \dagger$ & 0.46 (0.34 to 0.61$)$ & 0.10 (0.04 to 0.27$)$ \\
\hline $\mathrm{HR}(95 \% \mathrm{Cl})$ & $0.42(0.26$ to 0.68$), p=0.0003$ & Reference (1.0) \\
\hline Median OS, months & 15.5 (10.3 to 25.1$)$ & 9.5 (7.6 to 21.3$)$ \\
\hline 6-month OS $(95 \% \mathrm{Cl}) \dagger$ & 0.71 (0.60 to 0.84$)$ & 0.73 (0.6 to 0.87$)$ \\
\hline 12-month OS $(95 \% \mathrm{Cl}) \dagger$ & $0.58(0.46$ to 0.73$)$ & 0.39 (0.26 to 0.57$)$ \\
\hline $\mathrm{HR}(95 \% \mathrm{Cl})$ & 0.62 (0.39 to 1.01$), p=0.053$ & Reference (1.0) \\
\hline
\end{tabular}

${ }^{*} \mathrm{P} / \mathrm{LP}$ show similar proportions and are combined.

†Proportion.

VUS, variants of unknown significance. 
Mutation + LP+P + VUS

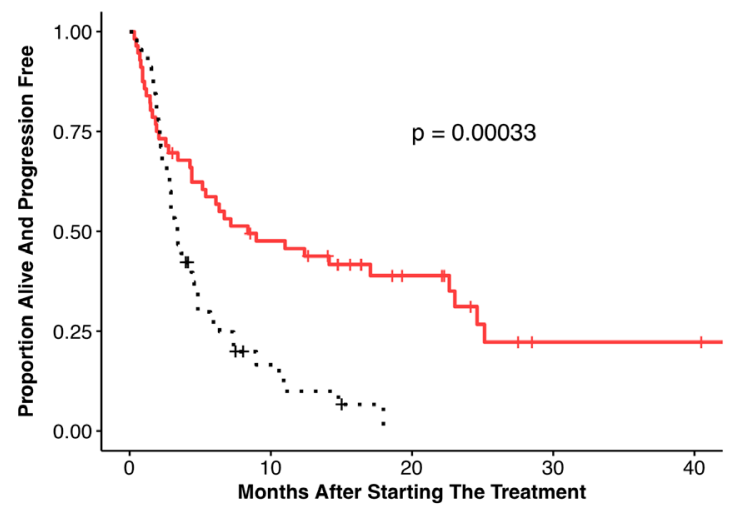

Number at risk

$\begin{array}{cccccc}\mathrm{LP}+\mathrm{P} & 56 & 25 & 12 & 3 & 3 \\ \text { VUS } & 45 & 5 & 0 & 0 & 0\end{array}$

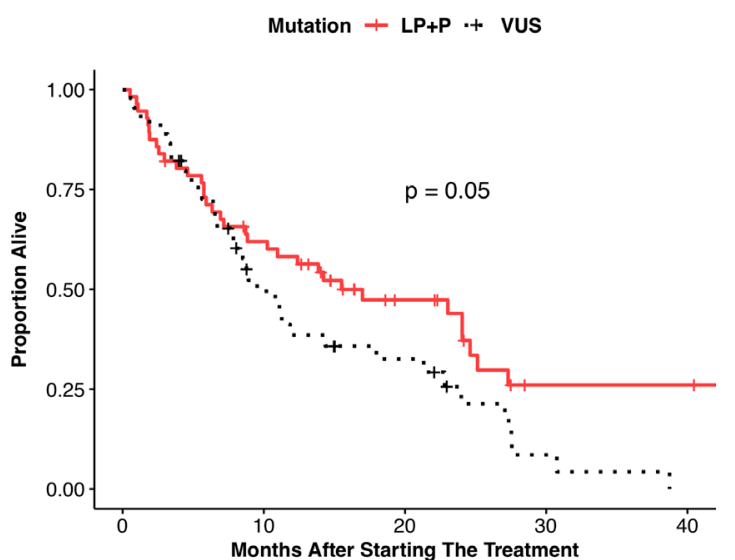

Number at risk

$\begin{array}{rrrrrr}\mathrm{LP}+\mathrm{P} & 56 & 33 & 16 & 5 & 5 \\ \text { Vus } & 45 & 18 & 10 & 2 & 0\end{array}$

Figure 3 Kaplan-Meier curves showing (A) progression-free survival and (B) overall survival for subjects with pathogenic or likely pathogenic $L R P 1 B$ alterations (P/LP) and variants of unknown significance (VUS). $\mathrm{P}$ values shown are determined from the log-rank test.

and remained consistent when adjusting for TMB and excluding MSI-H (data not shown).

\section{Extraordinary responder analysis}

There were 24 out of $56(42.9 \%)$ patients free from progression or death for 12 months after initiating ICI therapy and having a $\mathrm{P} / \mathrm{LP}$ alteration in $L R P 1 B$. This compares to only 3 out of $45(6.7 \%)$ patients with $L R P 1 B$ VUS alterations who were free from progression or death after 12 months. Of those with pathogenic alterations in $L R P 1 B, 10$ patients had NSCLC with 4 having PD-L1 expression $>1 \%(60 \%, 80 \%, 95 \%$ and $>1 \%)$ and 5 having $\mathrm{TMB}>10$ mut/Mb (12, 21.7, 31, 66.8 and 73 mut/Mb). Four patients with prostate cancer were identified (3 of 4 were MSI-H), and four patients with melanoma were identified (with TMBs of 8, 33, 62 and 107 mut/Mb). The other extraordinary responder cases included two with cutaneous SCC (TMB 87, 112 mut/Mb), one with esophageal cancer (TMB $116 \mathrm{mut} / \mathrm{Mb})$, two with unknown primary (TMB 43, 92 mut/Mb) and one with clear-cell kidney cancer. Of the three exceptional responders in the VUS group, two had lung cancer (TMB 9 and PD-L1 95\%; TMB 24.8 and PD-L1 unavailable) and one had colon cancer (microsatellite stable).

\section{DISCUSSION}

We performed the largest multicenter retrospective chart review of patients with $L R P 1 B$ alterations across multiple tumor type to our knowledge and found that patients with $\mathrm{P} / \mathrm{LP}$ alterations in $L R P 1 B$ have a higher ORR, improved PFS and improved OS compared with patients with $L R P 1 B$ VUS when treated with immune checkpoint blockade. Our results are suggestive that P/LP LRP1B alterations may be a tumor-agnostic biomarker-one which may help predict more favorable outcomes with ICIs, particularly PD-1 pathway blockade. A strength of our study is that our findings were consistent across all three institutions and in adjusted analyses for TMB and excluding MSI-H disease. Larger prospective studies are needed for external validation, but these results suggest that $L R P 1 B$ has clinical significance and should be included in targeted gene panels.

These results are supported by prior studies that have suggested that $L R P 1 B$ alterations may be associated with improved outcomes with ICI. In 2016, Johnson et alfound that alterations in $L R P 1 B$ was associated with high TMB and significantly associated with response to ICI therapy in patients with metastatic melanoma, being present in $11 / 32(34 \%)$ of responders compared with $1 / 33(3 \%)$ in non-responders $(\mathrm{p}<0.001) .{ }^{31}$ A more recent study found that OS was better among patients with NSCLC and melanoma with $L R P 1 B$ mutations compared with $L R P 1 B$ wildtype tumors. ${ }^{32}$ They also found a significantly higher TMB rate among $L R P 1 B$-mutated compared with $L R P 1 B$ wildtype tumors. Additionally, three case reports of patients having had unexpected responses to ICI also reported alterations in $L R P 1 B$ in human papillomavirus-related small cell cancer of the head and neck, sebaceous carcinoma and renal cell carcinoma with rhabdoid features. ${ }^{33-35}$ Finally, a recent study from our group reporting outcomes among men with metastatic castration-resistant prostate cancer found that $3 / 4(75 \%)$ patients with $L R P 1 B$ alterations experienced durable prostate-specific antigen (PSA) declines of $>50 \%$ as compared with $2 / 14(14.2 \%)$ of patients with $L R P 1 B$ wild-type cancer. ${ }^{36}$

A major unanswered question is the mechanism by which $L R P 1 B$ inactivation might modulate response to ICI. Its function both in normal tissue and its role in cancer is poorly understood. Reconciling LRP1B's role as a tumor suppressor ${ }^{14}$ with its immunomodulatory properties is difficult as one would not expect a tumor suppressor to affect immune evasion, or antigen presentation; however, $L R P 1 B$ 's role as a tumor suppressor has not been definitively established. There is evidence that 

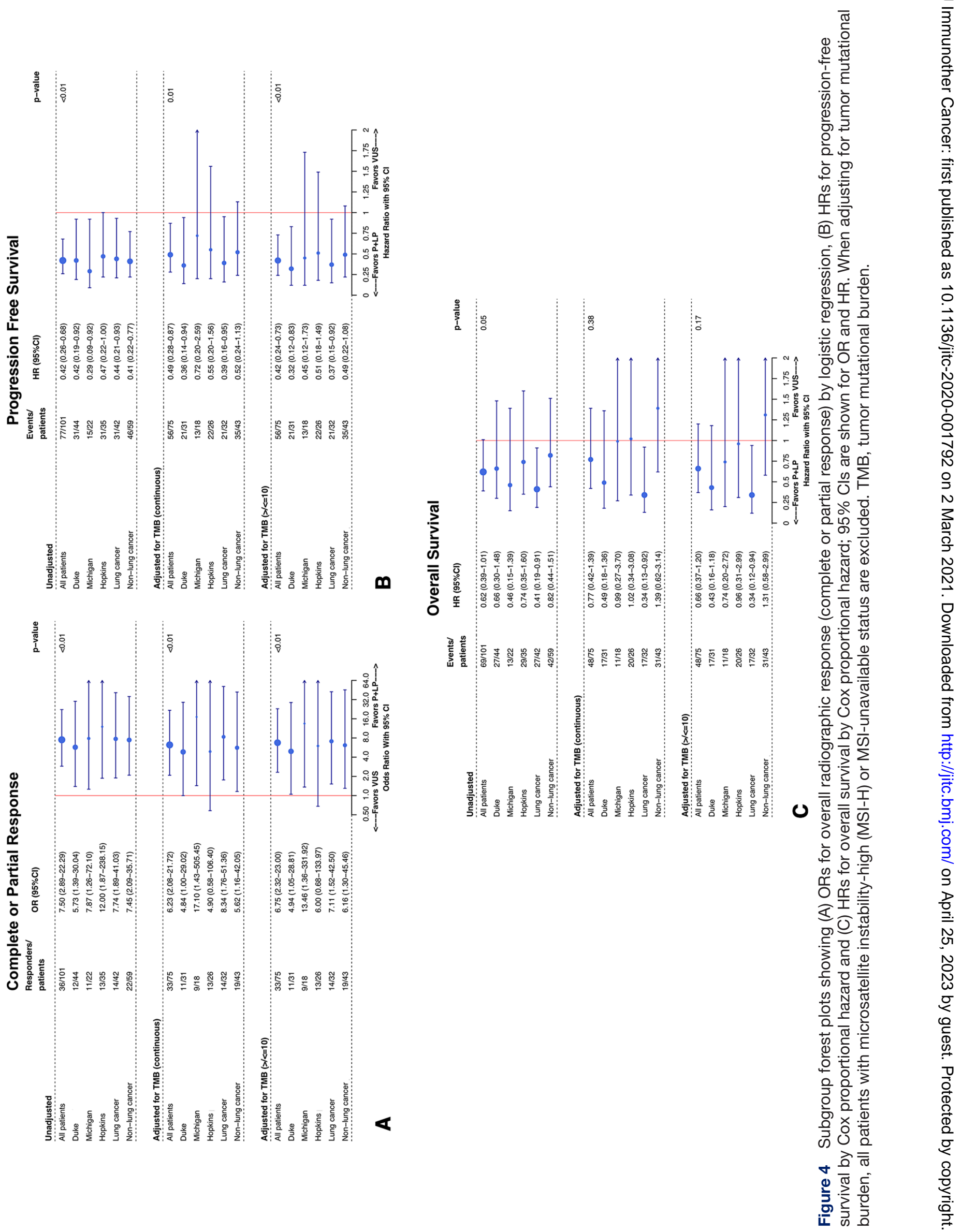
$L R P 1 B$ may participate in extracellular ligand scavenging through its role as an endocytic receptor, thereby modulating the tumor microenvironment ${ }^{23} 42$ or could participate in cellular drug uptake. ${ }^{43}$ One previous study found that cell cycle and antigen processing pathways were significantly altered in tumors with $L R P 1 B$ alterations, and patients with $L R P 1 B$ mutations had higher T-cell inflamed gene expression scores ${ }^{32}$ Finally, could $L R P 1 B$ impact the immune cell infiltrate and therefore a functional $L R P 1 B$ could diminish immune recognition of a malignant cell? Only further mechanistic studies in immunocompetent preclinical models of lung and other cancer subtypes treated with ICI therapy will address this functional relevance and mechanisms of immune recognition.

Conversely, there is evidence that $L R P 1 B$ may be associated with a higher TMB given its large size as well as its location at the common fragile site, FRA2F. ${ }^{44}$ Our study did not note a statistical difference in TMB between the two $L R P 1 B$ cohorts, although there were more outliers with $\mathrm{TMB}>50 \mathrm{mut} / \mathrm{Mb}$ in the $\mathrm{P} / \mathrm{LP}$ group compared with the VUS group. A strength of our study is the comparison between outcomes of patients with pathogenic $L R P 1 B$ alterations with those with $L R P 1 B$ VUS to reduce confounding from TMB. Furthermore, our post hoc analysis excluding MSI-H cases and adjusting for TMB suggests that $L R P 1 B$ may have independent predictive value beyond that which is mediated by TMB. Despite this, we do recognize that the lack of comparison to outcomes among patients with $L R P 1 B$ wild-type malignancies is a significant shortcoming and limits its interpretation. Thus, prospective pan-cancer studies are needed comparing outcomes with ICI-treated patients with and without $L R P 1 \mathrm{~B}$ alterations.

Our study included a diverse set of malignancies. Interestingly, we found that with regard to overall response and PFS, the improved outcomes in the P/LP group over the VUS group were consistent for both patients with lung cancer (the largest subset) and patients with non-lung cancers. However, the improved OS in the entire cohort appeared to be primarily driven by the patients with lung cancer. Given its high incidence as well as the frequency of $L R P 1 B$ alterations, these findings require further investigation, specifically in lung cancer. Although ICI is part of the standard-of-care first-line regimen for all patients with NSCLC without a driver mutation (such as EGFR, $A L K$ or ROS1), $L R P 1 B$ alterations may identify a subpopulation of patients with lower PD-L1 expression who can be treated with ICI monotherapy. The size of our cohort limits our ability to make any inferences about specific tumor types other than lung cancer, but $L R P 1 B$ potentially remains an intriguing tumor-agnostic biomarker candidate across multiple tumor types.

This study has several additional limitations. First, this is a small, retrospective study conducted entirely at academic institutions where many patients had been heavily pretreated prior to receiving immune checkpoint inhibition. Additionally, the only patients eligible were those who had tissue-based NGS testing. Additionally, given our limited understanding of the normal function of $L R P 1 B$, the definition of $\mathrm{P} / \mathrm{LP}$ or VUS alterations may be problematic. We are particularly cautious about interpretations of this biomarker because this is not well defined. We took a prespecified systematic approach to our analysis to define the patient subgroups, but it is challenging to determine whether a detected genomic alteration is truly 'pathogenic'. This definition will likely require further refinement over time with better understanding of $L R P 1 B$ 's role in normal tissue and cancer. ICI therapy was not standardized across patients and the asymmetry between the distribution in tumor types across the $L R P 1 B$ subgroups raises concerns over unmeasured confounders. Of note, there is a higher rate of anti-CTLA-4 therapy in the P/LP group compared with the VUS group, although the improvements in ORR, PFS and OS remained consistent even after excluding the nine patients who had received anti-CTLA-4 therapy from the analysis. As mentioned above, the choice of a control group is also a limitation. LRP1B VUS was chosen as a convenience control group, but no inferences can be made in comparison to patients with $L R P 1 B$ wild-type malignancies. Wild-type malignancies were not used as a control group due to the challenges of appropriate controlling and matching leading to increased heterogeneity. Finally, although not a direct limitation on this analysis, $L R P 1 B$ is no longer included in the current version of the FoundationOne CDX panel, which will limit its study and prospective validation moving forward. $L R P 1 B$ is also not included on Guardant360, FoundationOneLiquid, or MSK-IMPACT panels, but is included on Caris Molecular Intelligence, Personal Genome Diagnostics and MI-ONCOSEQ panels.

In summary, in our multicenter, retrospective cohort study of patients with multiple tumor types, $L R P 1 B \mathrm{P} / \mathrm{LP}$ alterations are associated with improved response rates, PFS and OS when treated with ICI compared with patients with $L R P 1 B$ VUS alterations. Our findings were strongest in patients with NSCLC and were independent of high TMB and MSI status. Further mechanistic studies into the function of $L R P 1 B$ are needed as well as prospective validation of this potential predictive clinical biomarker for ICI responses.

\section{Author affiliations \\ ${ }^{1}$ Duke Cancer Institute Center for Prostate and Urologic Cancers, Durham, North Carolina, USA \\ ${ }^{2}$ Internal Medicine, Division of Hematology and Oncology, Vanderbilt University Medical Center, Nashville, Tennessee, USA \\ ${ }^{3}$ Johns Hopkins Medicine Sidney Kimmel Comprehensive Cancer Center, Baltimore, Maryland, USA \\ ${ }^{4}$ Division of Hematology Oncology, Department of Internal Medicine, University of Michigan, Ann Arbor, Michigan, USA \\ ${ }^{5}$ Levine Cancer Institute, Charlotte, North Carolina, USA \\ ${ }^{6}$ Biostatistics and Bioinformatics, Duke University, Durham, North Carolina, USA}

Twitter Matthew K Labriola @MattLabs831

Acknowledgements The authors would like to thank the patients and staff at the Duke Cancer Institute, Sidney Kimmel Comprehensive Cancer Center and Rogel Cancer Center. 
Contributors LCB: writing — original draft, investigation, formal analysis. MDT, RS, EBS, CK, MKL, RTG, DM: investigation. JZ: conceptualization, investigation. SG, YW: formal analysis. TZ, MH, DG: writing—review and editing. AA, EA: supervision, writing —review and editing. AJA: conceptualization, supervision, writing—review and editing.

Funding This research received no external funding. AJA is supported by the following federal funding: NIH P30 CA014236, NIH R01 1R01CA233585-01. EA is supported by NIH grant P30 CA006973 and DOD grant W81XWH-16-PCRP-CCRSA.

Competing interests JZ: consulting: NGM Biopharmaceuticals, UroToday; Travel Expenses: UroToday. RTG: speakers bureau: Bayer Pharma AG; Consulting Fees: Bayer Pharma AG, Invivo Corp, Bard. TZ: research funding: AstraZeneca, Janssen, OmniSeq, PGDx, Pfizer, Merrimack, AbbVie/Stemcentrx, Novartis, Merck, Mirati and Regeneron; Advisory/consultant role: Genentech Roche, Exelixis, Bayer, AstraZeneca, Pfizer, Sanofi-Aventis, Janssen, Foundation Medicine, Amgen, Bristol-Myers Squibb, Merck, Pharmacyclics and Seattle Genetics; speakers bureau: Genentech Roche, Exelixis, Sanofi-Aventis, Genomic Health; Stocks/ Employment: Capio Biosciences (spouse), Archimmune Therapeutics (spouse). MH: consultant: AstraZeneca, Bayer, Bristol-Myers Squibb, Exelixis, Genentech, Janssen, Pfizer; speakers bureau: Exelixis and Genentech; Research Funding (to institution): Acerta, Bristol-Myers Squibb, Clovis, Exelixis, Genentech, Merck, Pfizer, Seattle Genetics. DG: Acerta Pharmaceuticals—Research, American Association for Cancer Research-Sr Editor, Astellas—Consultant, Research, Advisory Board, AstraZeneca-Consultant, Advisory Board, Axess Oncology_-Independent Contractor, Bayer H/C Pharmaceuticals-Research, Consultant, Speaker, Honorarium, Travel accommodations, SC, Bristol-Myers Squibb-Consultant, Research, Steering Committee, Calithera-Research, Capio Biosciences—Scientific Advisory Board, EMD Serono-Honorarium, Exelixis, Inc-Research, Consultant, Speaker, Honorarium, Travel accommodations, Flatiron-Consultant, IpsenHonorarium, Janssen Pharmaceuticals—Research, Consultant, Independent Data Monitoring Committee (IDMC), Leidos Biomedical Research—Consultant, Merck Sharp \& Dohme - Consultant, Michael J Hennessey Associates - Honorarium, Consultant, Millennium Medical Publishing, Clinical Advances in Hematology \& Oncology - Co-Editor-in-Chief, Modra Pharmaceuticals-Advisory Board, Myovant Sciences, Inc-Consultant, Nektar Therapeutics-Steering Committee, NovartisResearch, Physician Education Resource LLC-Consultant, Pfizer-Research, Consultant, Steering Committee, Honorarium, Sanofi-Research, Consultant, Speaker, Honorarium, Travel accommodations, UroGPO—Honorarium, UroTodayHonorarium, Travel accommodations, Vizuri Health Sciences, LLC—Consultant, $\mathrm{NCl}$-Steering Committee. AJA: consultant and/or advisor for AstraZeneca; Bristol-Myers Squibb; Merck \& Co; Pfizer, EMD Serono. Paid DSMB member: Eisai. Grant/Research Support from AstraZeneca; Bristol-Myers Squibb and Merck \& Co. Other financial or material support from Arcus Biosciences; Astellas Pharma US; Celgene Corporation; Clovis Oncology; Pfizer; Prometheus Biosciences and Seattle Genetics in the form of research support to institution. EA: grants and personal fees from Janssen, personal fees from Astellas, grants and personal fees from Sanofi, grants and personal fees from Dendreon, personal fees from Pfizer, personal fees from Invitae, grants and personal fees from AstraZeneca, grants and personal fees from Clovis, grants and personal fees from Merck, grants from Johnson \& Johnson, grants from Genentech, grants from Novartis, grants from Bristol Myers-Squibb; and a patent (PCT/US2015/046806; US20170275673A1) on an AR-V7 biomarker technology that is licensed to Qiagen. AJA: consulting fees: AstraZeneca, Merck, Dendreon, Janssen, Clovis, Bayer and Medivation/Astellas; speaking fees: Bayer and Dendreon, Research funding to Duke: Janssen, Medivation/Astellas, SanofiAventis, Active Biotech, Bayer, Dendreon, Merck, AstraZeneca, Genentech/Roche, Bristol-Myers Squibb, Constellation, Novartis and Pfizer.

Patient consent for publication Not required.

Provenance and peer review Not commissioned; externally peer reviewed.

Data availability statement Data are available on reasonable request.

Open access This is an open access article distributed in accordance with the Creative Commons Attribution Non Commercial (CC BY-NC 4.0) license, which permits others to distribute, remix, adapt, build upon this work non-commercially, and license their derivative works on different terms, provided the original work is properly cited, appropriate credit is given, any changes made indicated, and the use is non-commercial. See http://creativecommons.org/licenses/by-nc/4.0/.

\section{ORCID iDs}

Landon C Brown http://orcid.org/0000-0002-5231-4859

Matthew D Tucker http://orcid.org/0000-0002-8193-8986

Matthew K Labriola http://orcid.org/0000-0003-0919-9864

Tian Zhang http://orcid.org/0000-0001-8914-3531

\section{REFERENCES}

1 Reck M, Rodríguez-Abreu D, Robinson AG, et al. Pembrolizumab versus chemotherapy for PD-L1-positive non-small-cell lung cancer. N Engl J Med 2016;375:1823-33.

2 Wolchok JD, Chiarion-Sileni V, Gonzalez R, et al. Overall survival with combined nivolumab and ipilimumab in advanced melanoma. $N$ Engl J Med 2017;377:1345-56.

3 Motzer RJ, Tannir NM, McDermott DF, et al. Nivolumab plus ipilimumab versus sunitinib in advanced renal-cell carcinoma. $N$ Engl J Med 2018;378:1277-90.

4 Le DT, Durham JN, Smith KN, et al. Mismatch repair deficiency predicts response of solid tumors to PD-1 blockade. Science 2017;357:409-13.

5 Marabelle A, Fakih MG, Lopez J, et al. Association of tumour mutational burden with outcomes in patients with select advanced solid tumours treated with pembrolizumab in KEYNOTE-158. Ann Oncol 2019;30:v477-8.

6 Hellmann MD, Callahan MK, Awad MM, et al. Tumor mutational burden and efficacy of nivolumab monotherapy and in combination with ipilimumab in small-cell lung cancer. Cancer Cell 2018;33:853-61.

7 Tumeh PC, Harview CL, Yearley JH, et al. PD-1 blockade induces responses by inhibiting adaptive immune resistance. Nature 2014;515:568-71.

8 Jiang P, Gu S, Pan D, et al. Signatures of T cell dysfunction and exclusion predict cancer immunotherapy response. Nat Med 2018;24:1550-8.

9 Ayers M, Lunceford J, Nebozhyn M, et al. IFN- $\gamma$-related mRNA profile predicts clinical response to PD-1 blockade. J Clin Invest 2017; 127:2930-40.

10 Chaput N, Lepage P, Coutzac C, et al. Baseline gut microbiota predicts clinical response and colitis in metastatic melanoma patients treated with ipilimumab. Ann Oncol 2017;28:1368-79.

11 Liu CX, Li Y, Obermoeller-McCormick LM, et al. The putative tumor suppressor LRP1B, a novel member of the low density lipoprotein (LDL) receptor family, exhibits both overlapping and distinct properties with the LDL receptor-related protein. J Biol Chem 2001;276:28889-96.

12 Liu CX, Musco S, Lisitsina NM, et al. Genomic organization of a new candidate tumor suppressor gene, LRP1B. Genomics 2000;69:271-4

13 Lu Y-J, Wu C-S, Li H-P, et al. Aberrant methylation impairs low density lipoprotein receptor-related protein 1B tumor suppressor function in gastric cancer. Genes Chromosomes Cancer 2010;49:412-24.

14 Beer AG, Zenzmaier C, Schreinlechner M, et al. Expression of a recombinant full-length LRP1B receptor in human non-small cell lung cancer cells confirms the postulated growth-suppressing function of this large LDL receptor family member. Oncotarget 2016;7:68721-33.

15 Langbein S, Szakacs O, Wilhelm M, et al. Alteration of the LRP1B gene region is associated with high grade of urothelial cancer. $L a b$ Invest 2002;82:639-43.

16 Pineau P, Marchio A, Nagamori S, et al. Homozygous deletion scanning in hepatobiliary tumor cell lines reveals alternative pathways for liver carcinogenesis. Hepatology 2003;37:852-61.

17 Sonoda I, Imoto I, Inoue J, et al. Frequent silencing of low density lipoprotein receptor-related protein 1B (LRP1B) expression by genetic and epigenetic mechanisms in esophageal squamous cell carcinoma. Cancer Res 2004;64:3741-7.

18 Hirai Y, Utsugi K, Takeshima N, et al. Putative gene loci associated with carcinogenesis and metastasis of endocervical adenocarcinomas of uterus determined by conventional and arraybased CGH. Am J Obstet Gynecol 2004;191:1173-82.

19 Roversi G, Pfundt R, Moroni RF, et al. Identification of novel genomic markers related to progression to glioblastoma through genomic profiling of 25 primary glioma cell lines. Oncogene 2006;25:1571-83.

20 Nakagawa T, Pimkhaokham A, Suzuki E, et al. Genetic or epigenetic silencing of low density lipoprotein receptor-related protein 1B expression in oral squamous cell carcinoma. Cancer Sci 2006;97:1070-4.

21 Rahmatpanah FB, Carstens S, Guo J, et al. Differential DNA methylation patterns of small B-cell lymphoma subclasses with different clinical behavior. Leukemia 2006;20:1855-62.

22 Taylor KH, Pena-Hernandez KE, Davis JW, et al. Large-Scale CpG methylation analysis identifies novel candidate genes and reveals methylation hotspots in acute lymphoblastic leukemia. Cancer Res 2007;67:2617-25.

23 Prazeres $\mathrm{H}$, Torres J, Rodrigues F, et al. Chromosomal, epigenetic and microRNA-mediated inactivation of LRP1B, a modulator of the extracellular environment of thyroid cancer cells. Oncogene 2011;30:1302-17. 
24 Nikolaev SI, Rimoldi D, Iseli C, et al. Exome sequencing identifies recurrent somatic MAP2K1 and MAP2K2 mutations in melanoma. Nat Genet 2011;44:133-9.

25 Cowin PA, George J, Fereday S, et al. LRP1B deletion in high-grade serous ovarian cancers is associated with acquired chemotherapy resistance to liposomal doxorubicin. Cancer Res 2012;72:4060-73.

$26 \mathrm{Ni} \mathrm{S}, \mathrm{Hu}$ J, Duan Y, et al. Down expression of LRP1B promotes cell migration via RhoA/Cdc42 pathway and actin cytoskeleton remodeling in renal cell cancer. Cancer Sci 2013;104:817-25.

27 Ross JS, Wang K, Rand JV, et al. Next-Generation sequencing of adrenocortical carcinoma reveals new routes to targeted therapies. $J$ Clin Pathol 2014;67:968-73.

28 Beroukhim R, Mermel CH, Porter D, et al. The landscape of somatic copy-number alteration across human cancers. Nature 2010;463:899-905.

29 Cerami E, Gao J, Dogrusoz U, et al. The cBio cancer genomics portal: an open platform for exploring multidimensional cancer genomics data. Cancer Discov 2012;2:401-4.

30 Gao J, Aksoy BA, Dogrusoz U, et al. Integrative analysis of complex cancer genomics and clinical profiles using the cBioPortal. Sci Signal 2013;6:pl1.

31 Johnson DB, Frampton GM, Rioth MJ, et al. Targeted next generation sequencing identifies markers of response to PD-1 blockade. Cancer Immunol Res 2016;4:959-67.

32 Chen $\mathrm{H}$, Chong $\mathrm{W}, \mathrm{Wu} \mathrm{Q}$, et al. Association of LRP1B Mutation With Tumor Mutation Burden and Outcomes in Melanoma and Nonsmall Cell Lung Cancer Patients Treated With Immune Check-Point Blockades. Front Immunol 2019;10:1113.

33 Ho WJ, Rooper L, Sagorsky S, et al. A robust response to combination immune checkpoint inhibitor therapy in HPV-related small cell cancer: a case report. J Immunother Cancer 2018;6:33.
34 Domingo-Musibay E, Murugan P, Giubellino A, et al. Near complete response to pembrolizumab in microsatellite-stable metastatic sebaceous carcinoma. J Immunother Cancer 2018;6:58.

35 Wynja E, Solomon B, Bleeker J. Complete and prolonged response of renal cell carcinoma with rhabdoid features to checkpoint inhibitor therapy. J Immunother 2018;41:340-2.

36 Tucker MD, Zhu J, Marin D, et al. Pembrolizumab in men with heavily treated metastatic castrate-resistant prostate cancer. Cancer Med 2019;8:4644-55.

37 Forbes SA, Beare D, Boutselakis $\mathrm{H}$, et al. COSMIC: somatic cancer genetics at high-resolution. Nucleic Acids Res 2017;45:D777-83.

38 Shihab HA, Rogers MF, Gough J, et al. An integrative approach to predicting the functional effects of non-coding and coding sequence variation. Bioinformatics 2015;31:1536-43.

39 Schwartz LH, Litière $S$, de Vries $E$, et al. RECIST 1.1-Update and clarification: from the RECIST Committee. Eur J Cancer 2016;62:132-7.

40 Hellmann MD, Paz-Ares L, Bernabe Caro R, et al. Nivolumab plus ipilimumab in advanced non-small-cell lung cancer. $N$ Engl J Med 2019;381:2020-31.

41 Hellmann MD, Ciuleanu T-E, Pluzanski A, et al. Nivolumab plus ipilimumab in lung cancer with a high tumor mutational burden. $N$ Engl J Med 2018;378:2093-104.

42 Dietrich MF, van der Weyden L, Prosser HM, et al. Ectodomains of the LDL receptor-related proteins LRP1b and LRP4 have anchorage independent functions in vivo. PLoS One 2010;5:e9960.

43 Chung NS, Wasan KM. Potential role of the low-density lipoprotein receptor family as mediators of cellular drug uptake. Adv Drug Deliv Rev 2004;56:1315-34.

44 Smith DI, Zhu Y, McAvoy S, et al. Common fragile sites, extremely large genes, neural development and cancer. Cancer Lett 2006;232:48-57. 